\title{
Socioeconomic Determinants of First Names
}

\author{
Gerrit Bloothooft and David ONLAND \\ Utrecht Institute of Linguistics, NL
}

Modern naming practices in the Netherlands between 1982 and 2005 were studied on the basis of 1409 popular first names, divided into fourteen name groups determined by the common preferences of parents for the names involved. Socioeconomic variables such as family income, parents' level of education, and lifestyle indicators were analyzed in relation to the names - and name groups - of the children in 281,751 households. Naming practices could be described on two independent dimensions. The first of these was education and family income: parents with lower incomes and levels of education preferred English, Italian, Spanish, and international names, while those with higher incomes and levels of education chose predominantly Dutch, Frisian, Nordic, Hebrew, and French names. A second dimension distinguished between conservative and religious parents with a preference for traditional names, and trendy parents who favored shorter and modern names. The complex nature of the relationship between social class and naming practice, and its dynamics, is discussed.

KEYWORDS first names, lifestyle, education, income, social classes

\section{Introduction}

Naming practices changed in a revolutionary way during the twentieth century in Western Europe. While choice of first names in previous centuries was predominantly bound by traditions that prescribed naming children after relatives, saints, or godparents (Leys, 1974; Seibicke, 1996), a diminishing role of the church, loss of tradition, urbanization, and emerging individualization created freedom for parents. This freedom allowed parents to follow their personal name preferences, which, however, were and still are influenced by social stratification and fashion. The diffusion of name innovations across social strata, which had followed a top-down direction for centuries, lost prominence as social classes developed their own preferences (Besnard \& Grange, 1993). Class-related naming preferences usually did not involve strict segregation in favored names, but rather a different distribution of popularity in each social class, with each group drawing from a large set of common names (Desplanques, 
I986). This observation is supported by Lieberson, who observed differences in naming practices between groups in Texas with different socioeconomic levels (Lieberson, 2000) and groups in New York with different education levels (Lieberson, I992). He concluded that there is little class imitation: different social classes adopt the same names if these come into fashion, but the turnaround is too fast to allow for top-down class imitation, even though adoption in higher social strata may in some cases be quicker. Fryer and Levitt (2004) describe a contrasting difference in naming practice between Blacks and Whites, with the growth of distinctively Black names following the Black Power movement in the early I970s in the USA. Thus, while changes in naming practice are evident throughout the western world over the last century, there may be considerable differences among and within countries, due to differences in driving mechanisms and speed of change, related to varying historical, cultural and religious conditions.

Leys (I974) noted that in West-Flanders, Belgium, it was the lower rural classes, rather than the elite, that started to adopt new and often foreign names in the I96os. He explains this as the result of contact with new name inventories through television. This reinforced the emerging freedom in naming practice for innovation's sake, which was not yet as widespread during the era of the radio. In earlier centuries, such freedom and contact with other cultures were privileges of the educated and higher social strata, albeit in a different way. The lower and middle classes now seem more eager than the elite to adopt new names that come to them through the popular media.

In Germany, Debus et al. (I973) investigated naming practices in Kiel for 7000 children born between 1958 and I966, and found a reduction in naming after relatives, a trend more pronounced for workers than for the elite. Gerhards (2003) compared naming during the whole of the twentieth century in the small towns of Gerolstein and Grimma, in the former Western and Eastern parts of Germany respectively. He found a faster rate of secularization in the Catholic Gerolstein, and a more gradual one (although with an earlier start in nineteenth century) in the Protestant Grimma. After differentiating parents on the basis of education required for their profession, he found that there were only minor differences between classes in the timing of the adoption of new names. Highly educated parents maintained Christian naming somewhat longer, while parents with intermediate education had the strongest preference for transnational names. He is unsure whether the generalization of Besnard and Grange (I993), that social classes in present-day France differ in their unique name choices, also holds for Germany. For Denmark, Andersen (I977) also concludes that the period I969-73 showed a marked increase in the choice of new names in the lower and middle classes, but he is hesitant to conclude that naming practices are class-related.

Desplanques (I986) investigated changes in naming practices on a firm statistical basis using a large sample (2.3 million in total) of names given in twentieth-century France, complemented by data on the professions of the parents. His pioneering analyses demonstrate that the transition from tradition-bound to modern naming practices took place relatively early in France, and had already begun in the second half of the nineteenth century. He concludes that, while each socioeconomic class developed a preference for certain types of first names, the vast majority of French 
names are found across all classes, albeit with different levels of popularity. He found that parents with higher education levels took the lead in the adoption of new French names, but the slowest adopters, rural farmers, lagged behind by only five years (whereas Besnard and Grange (I993) showed that a century ago the elite were about thirty years ahead in naming trends). It remains to be seen whether the same holds for the adoption of, for instance, Anglo-American names.

In the Netherlands, differences in naming practices were long dominated by religion rather than social status. For official registration, Catholics predominantly chose Latinized names (for instance, Wilhelmus), and Protestants chose similar names but with Dutch spelling (Willem), or names of Germanic or Frisian origin. Differences between the groups in the use of familiar names, which were usually abbreviated forms of official names, were less distinctive, especially for males. This relatively stable situation changed dramatically during the twentieth century, which is demonstrated by the sudden decline in popularity after I950 of Maria, once the most popular name (see Figure I). In France, Marie shows the same tendency, but with an even steeper decline from its height at 34 percent, starting at the end of the nineteenth century (Dupâquier et al., I986). Among Protestants in the Dutch population, a gradual decline in naming children after relatives also started in the nineteenth century, as demonstrated by the occurrence of the name Jan (Figure 2). This development compares to the observations of Gerhards (2003) in Germany.

The more or less stable frequency of the traditional names in the Netherlands around the beginning of the twenty-first century indicates that the transition that started a century ago from traditional naming after relatives to free choices for parents has been completed. This by no means implies that naming trends have stabilized. On the contrary, they are more dynamic than ever with names coming and going continuously. Under these new naming practices, Bloothooft and Groot (2008) studied the first names of all 4.5 million children born in the Netherlands between I983 and 2005. They analyzed the co-occurrence of names of siblings, and grouped names that were often found in families. For instance, Maria and Johannes are often

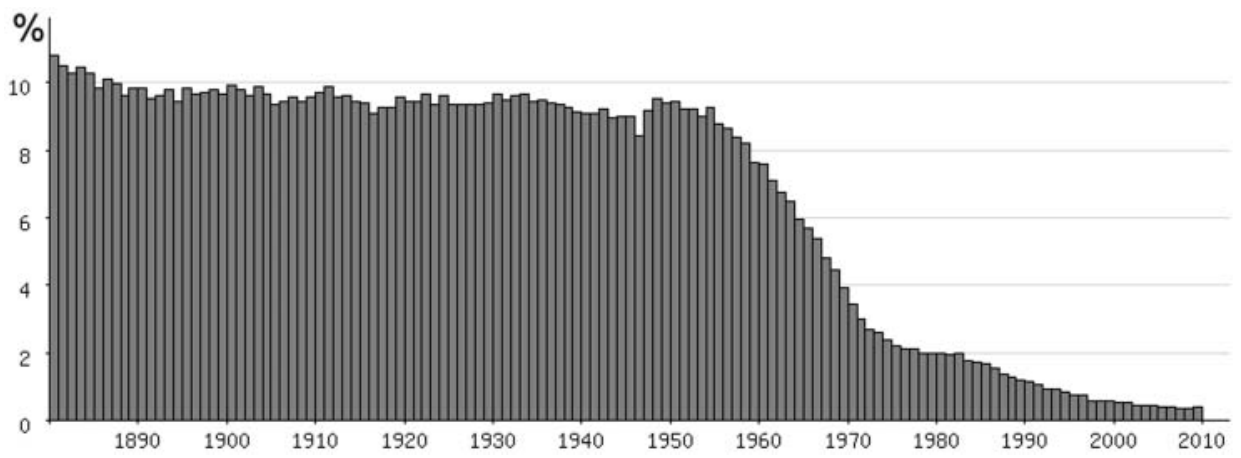

FIGURE 1 Reduction of the traditional naming practice between 1880 and 2009, exemplified by the reduced relative popularity of the Catholic name Maria, with a sudden fall between 1955 and 1975. Popularity distributions for all first names in the Netherlands can be found at www.voornamenbank.nl. 


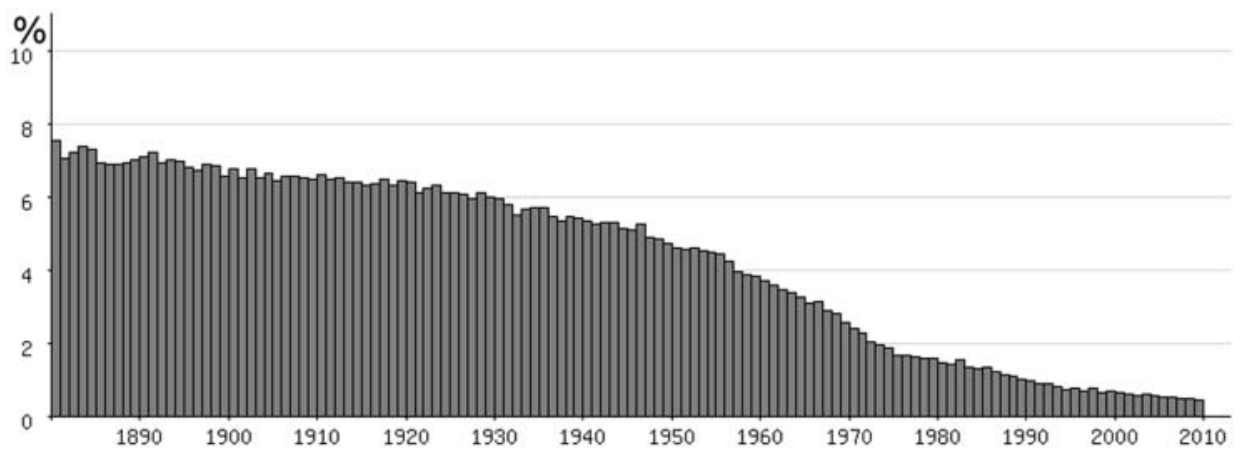

FIGURE 2 The gradual decline of the relative popularity of the male Protestant name Jan.

found together in a family, as are Kevin and Kimberley, and Mohamed and Fatima, while this is not the case for Kevin and Maria or Mohamed and Johannes. They used this analysis to divide the I409 most frequent names into 34 distinct groups (see Table I), each of which is related to a common preference of the respective parents. The name groups typically have an easily observable common denominator, such as language origin (for example, Dutch, Frisian, French, English, Nordic, Arabic, or Turkish names), religion (biblical and Latinized names), period of highest popularity (traditional to modern), gender (given to boys or girls only), or morphology (suffix type and length).

Our assumption is that parents who share a preference for certain names for their children do so on the basis of a common socioeconomic, cultural, ethnic, or linguistic background. The last three factors are jointly known as CEL (cultural - ethnic linguistic) factors. Mateos, Webber, and Longley (2007) categorized the entire population of Great Britain in I85 CEL-types (a subdivision of I5 CEL-groups) based on a combination of techniques, including geographic spread. Because they did not include socioeconomic factors, their analysis resulted in CEL-types that are mainly based on ethnicity with little to no subdivision in social strata of the major categories. The largest CEL type, of English names, therefore included 3I.I million people, 68 percent of the total.

An investigation into the socioeconomic determinants of naming practices could start by defining the social strata into which parents are to be classified, while subsequently investigating the naming characteristics in each class. However, defining social strata is commonly identified as a major problem. Our study approaches the issue from the other direction. We take name groups as a starting point, under the assumption that each group is associated to coherent socioeconomic or lifestyle characteristics of the parents; our study attempts to clarify this association.

\section{Data and analysis}

We analyzed the names of children in households for which social and economic data were known. Although this kind of detailed information can be difficult to obtain due to privacy concerns, we were able to use data from a large private survey by a direct marketing company on consumption and personal backgrounds. De Grote 
TABLE 1

FOURTEEN MAIN NAME GROUPS RELATED TO THE THIRTY-FOUR NAME GROUPS IDENTIFIED IN BLOOTHOOFT \& GROOT (2008), WITH NAME EXAMPLES AND THE TOTAL NUMBER OF CHILDREN BORN BETWEEN 1982 AND 2005, AND OF THOSE MENTIONED IN THE WDM SURVEY

\begin{tabular}{|c|c|c|c|c|}
\hline Main group & $\begin{array}{l}\text { Description of initial } \\
\text { name groups }\end{array}$ & Examples (female, male) & $\begin{array}{c}\text { Number } \\
1982-2005\end{array}$ & $\begin{array}{c}\text { Number in } \\
\text { survey }\end{array}$ \\
\hline \multirow[t]{2}{*}{ Dutch-Traditional } & Latin form & Maria, Johannes & 187,133 & 2152 \\
\hline & Dutch form & Aaltje, Willem & 248,803 & 10,986 \\
\hline Frisian & Frisian & Femke, lelle & 100,871 & 10,725 \\
\hline \multirow[t]{3}{*}{ Elite } & Upcoming & Charlotte, Floris & 187,071 & 17,312 \\
\hline & Declining & Liselotte, Roderick & 13,662 & 867 \\
\hline & Group Alexander & Barbara, Alexander & 33,122 & 1897 \\
\hline Hebrew & Hebrew & Esther, Daniël & 152,291 & 14,550 \\
\hline \multirow[t]{3}{*}{ Dutch-preModern } & International & Laura, Mark & 250,732 & 35,430 \\
\hline & Group Thomas & Eline, Thomas & 343,881 & 37,846 \\
\hline & Group Eric & 一, Eric & 9953 & 1440 \\
\hline Dutch-Modern & Dutch modern & Sanne, Tim & 575,780 & 73,376 \\
\hline \multirow[t]{5}{*}{ English } & Premodern & Kimberley, Kevin & 759,960 & 106,169 \\
\hline & Upcoming short & Kim,Nick & 133,924 & 20,666 \\
\hline & $y$-suffix & Kelly, Davy & 61,863 & 9262 \\
\hline & Royal names & 一, William & 2966 & 500 \\
\hline & Group Cheyenne & Cheyenne, Jermaine & 5372 & 771 \\
\hline \multirow[t]{2}{*}{ French } & French & Maxime, Thierry & 19,459 & 2016 \\
\hline & Short & Beau, Jules & 33,234 & 2397 \\
\hline \multirow[t]{3}{*}{ Mixed(Nordic) } & Short & Bente, Mats & 17,557 & 2044 \\
\hline & Nordic and French & Anouk, Niels & 108,484 & 14,209 \\
\hline & Mixed names & Ingeborg, Lucas & 17,046 & 1724 \\
\hline \multirow[t]{2}{*}{ Modern } & Group Mika & Puck, Mika & 14,964 & 1607 \\
\hline & Group Milan & Zoë, Milan & 58,573 & 7743 \\
\hline Italian-Spanish & Italian and Spanish & Alicia, Lorenzo & 30,345 & 3854 \\
\hline Arabic1 & Group Mohamed & Fatima, Mohamed & 39,980 & 665 \\
\hline \multirow[t]{2}{*}{ Arabic2 } & Group Samir & Nadia, Samir & 17,447 & 719 \\
\hline & Group Yassine & Youssra, Yassine & 30,203 & 710 \\
\hline Turkish & Turkish & Merve, Ibrahim & 20,107 & 559 \\
\hline excluded & Mixed names & 一, Remko & 2408 & 398 \\
\hline excluded & Turkish, group Esra & Esra, Emre & 7127 & 237 \\
\hline excluded & Arabic, group Tarik & Ikram,Tarik & 9740 & 217 \\
\hline excluded & Arabic, group Hicham & Yasmina, Hicham & 8785 & 189 \\
\hline excluded & Slavic & Ivana, Igor & 1794 & 186 \\
\hline excluded & Italian, group Louisa & Louisa, Leonard & 1830 & 101 \\
\hline
\end{tabular}


Consumenten Enquête (lit. "big consumer survey") is a large biannual survey conducted by the WDM company in the Netherlands since I994. It consists of over seventy written questions that include, since 2002, the first name and date of birth of all household members. Ten surveys (2002-07) were used to create a comprehensive database of I.I3 million households (some I7 percent of all Dutch households). Because we were interested in households with children born after I982, only 28I,75 I households, containing altogether 5 I2,545 children, were usable. Of these, 383,533 children had one of the I409 first names that constitute the 34 name groups earlier identified by Bloothooft and Groot (2008), and were subjected to further study (see Table I). They constitute about Io percent of the total number of children born in the Netherlands in the period I982-2005.

From the abundance of data in the WDM survey, three factors were chosen as major socioeconomic determinants: family income, the highest education level of the parents, and the lifestyle of the household. The last factor was specified in some more detail through the likelihood of attending classical concerts, pop concerts, or religious services.

The WDM survey grouped family income before taxes into seven classes, defined relatively to the national average income. Participants were asked to choose between unknown, minimum, below average, average, $\mathrm{I} .5 \mathrm{x}, 2 \mathrm{x}, 2.5 \mathrm{x}$ and higher than $3 \mathrm{x}$ the average income. Given the likely uncertainty of some participants about their own gross income and/or the national average, we expect these answers to be indicative at best.

Information on the highest education level of the parents is likely to be far more accurate.

We classified the various education levels by total number of years of education (without internships), starting at the age of four years. The eight possible categories were: unknown, primary school (8 years of education), lower secondary vocational education (I I years), lower secondary school (I2 years), senior secondary vocational education (I4 years), higher secondary school (I4 years), higher vocational education (I7 years), and university (I8+ years). Due to the nature of the Dutch education system, there is no simple linear progression between the different categories, but total numbers of years of education is nevertheless indicative of level.

WDM distinguishes twenty lifestyles which can be attributed to a household on the basis of a weighting of all available data in the survey. Every household receives a score on each of the lifestyles. Although WDM did not inform us about the details of their analysis, we considered these lifestyle loadings a valuable parameter. To determine the indicators of lifestyle more explicitly — apart from income and education - we considered the attendance of classical concerts, pop concerts and religious services as examples of underlying determinants. Participants indicated whether they attended these events by a simple yes/no response. These figures were then converted into a probability per name group.

The participants in the WDM survey are volunteers, and therefore form a biased sample of the Dutch population. It is likely that households from the lowest and highest social strata are underrepresented. Therefore, we compared name groups only by looking at the relative differences within income and education classes, rather than comparing absolute figures. 
Also underrepresented in our sample are the Arabic and Turkish names, and the traditional Dutch names in their Latinized and traditional Dutch forms (see Table I). The likely explanation for the latter is that the Bloothooft and Groot (2008) analysis used only officially registered names, while the questionnaire respondents probably provided familiar names. For modern names, there is usually no difference between the official name and the familiar name, but for traditional names there often is (compare the official Franciscus with the abbreviated form Frans as a familiar name, or Margaretha with Grietje). By studying percentages across income and education classes, the bias of the sample can be overcome.

In our household sample, six name groups had a total frequency under 500 children. We decided to exclude these name groups from further analysis. Furthermore, in a preliminary analysis, we were able to determine that for some name groups with a common denominator, such as language, socioeconomic factors were highly similar. For reasons of simplicity and clarity of presentation, therefore, we decided to combine the twenty-eight remaining groups into fourteen main name groups. This summary process, including name examples, is presented in Table I. Below, we refer to these main name groups as name groups.

For our analyses, we needed parameter values (for instance, percentage higher education) for a name group. To this end, responses on the questionnaire were associated with all children in a specific family. These values were counted and averaged over all children with a name from a name group.

\section{Results and discussion}

\section{Income}

First, we investigated the distribution of household income for each name group. The resulting series of histograms are presented in a single plot in Figure 3, grouped

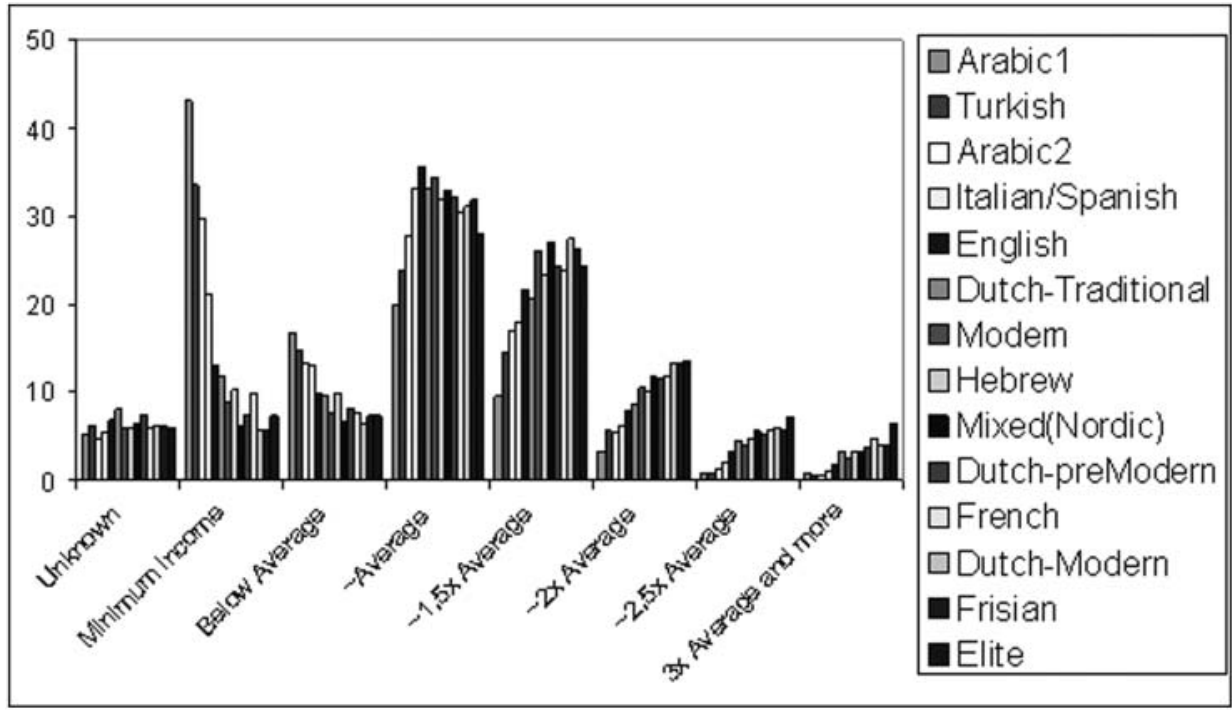

FIGURE 3 Combined presentation for all name groups of the distribution of households over income classes (in \% of the name group). 
by income class. The name groups within an income class are presented so that a gradual shift across name groups (increasing or decreasing) is evident in most classes. The order of presentation of the name groups is thus of interest. The series begins on the left with Arabic and Turkish name groups, followed Italian/Spanish and English name groups. These names are relatively frequently given by parents with low income, and much less often by parents with the highest income. The two Arabic and the Turkish name groups are special, in that the parents are probably following the traditions of their native language and culture, and form CEL groups that should be viewed separately from the mainstream. It should be noted that socioeconomic differentiation is evident within the Arabic CEL group, in the relatively higher presence of names from the Arabicz group in higher income categories. Italian/ Spanish or English names are chosen not primarily by the relatively small number of immigrants with these cultural origins, but rather by Dutch parents with an average income. The Italian/Spanish and English name groups, unlike the Arabic and Turkish name groups, do not emerge from CEL differentiation in the Netherlands. In this, they are comparable to the remaining name groups.

The series continues with groups of names of Dutch, Frisian, Hebrew, Nordic, and French origin and a group with modern non-Dutch names of various origins. The Dutch names are divided into traditional, pre-modern, and modern groups, which reflects their periods of maximum popularity: traditional names before 1960, premodern between I960 and I990, and modern after 1990. The traditional Dutch names, as well as Hebrew names from the Old Testament which also have a long history of use, tend to associate relatively more to middle incomes. They share this position with the modern names. The other Dutch, Nordic, French, and Frisian name groups are all somewhat stronger associated to incomes above average. The elite names (consisting of names connected to Dutch culture and history, and some French names, as French was once the preferred language of the nobility and the highest social classes) conclude the series with the relatively highest percentages found at the top incomes.

It should again be noted that our household sample does not necessarily reflect the Dutch population in all aspects. This means that the percentage of parents in some income classes may deviate from national figures. A comparison with an unbiased sample from the National Bureau of Statistics (2007; 7000 households) showed that the average-income class is overrepresented in the WDM survey, at the expense of the high- and low-income classes. Within an income class, however, we can study the relations among name groups without bias. Moreover, although the total number of households associated with a name group may vary greatly, this is normalized by presenting the relative distribution of a name group over income classes (in percentage). Despite the fact that we should be cautious in comparing percentages between income classes, it is clear that there is not a one-to-one relation between a name group and an income class. Since their fuzzy relationship could originate in the uncertainty of the income data, we will now look into the more accurate data of educational background.

\section{Education}

Figure 4 presents the distribution of households per name group over education categories. As already mentioned, there is no precise linear progression in the categories; 


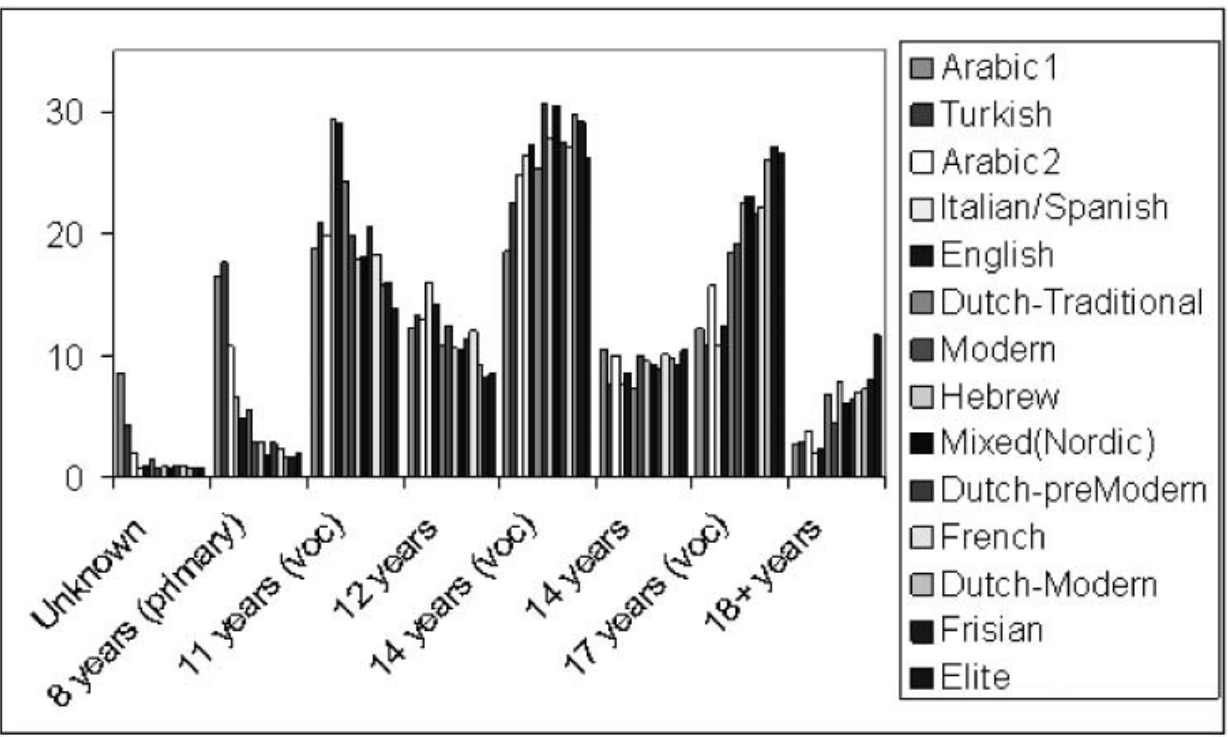

FIGURE 4 Combined presentation for all name groups of the distribution of households over maximum number of years of education of the parents (in \% of the name group). Education starts in the Netherlands at the age of four.

the overall tendency from left to right is one from low to high level of education. The clearer accuracy of these data as compared to the data on family income is exemplified by the lower percentages for the category "unknown." Furthermore, the overall results per education category match the results of an unbiased sample from the National Bureau of Statistics (2007; II,000 households) better than the income figures do; the education data show a maximum absolute deviation per category of less than 4 percent, with the exception of lower secondary vocational education or lower secondary education which are ro percent points higher.

The histograms are ordered by name group for education classes in the same way as for the income classes (above). This presentation facilitates a comparison of Figures 3 and 4 . Overall, gradual changes similar to those for income classes can be seen: starting with the Arabic, Turkish, Italian/Spanish, and English name groups, followed by the various Dutch, Hebrew, Nordic, French, and Frisian groups, while the elite name group appears at the far right of the range. This demonstrates the preference of parents with less education for Italian/Spanish and English names. Other name groups dominate the higher educational categories; we have already discussed the special position of the Arabic and Turkish name groups as specific CEL groups.

In addition, it should be mentioned that in the Netherlands education is compulsory until the age of seventeen (implying thirteen years of education), and children do not quit school after completing primary education. The high percentage of parents from the Arabic and Turkish name groups who report having completed only primary education therefore most likely represent first generation immigrants.

Alongside the relatively high positions of English and Italian/Spanish name group categories, the traditional Dutch name group also scores quite high in the lower 
secondary vocational education category. This suggests that Dutch parents with a lower level of education either uphold the tradition of naming after relatives (with, for instance, Dirk and Johanna) or switch to English and Italian/Spanish names (such as Dennis and Jennifer). They do not typically choose short Dutch names like Anne or Daan.

To illustrate the spread of names across educational categories, Table 2 shows the top twenty names for each. The highest rank order of a name is given in bold typeface. Many names can be found in several classes, with the widest diffusion apparent for the top names in the middle categories: Tim, Kevin, and Rick. Note the full segregation between the primary school level and the university level list: they do not share a single name in the top twenty. Because the top twenty list only shows the most popular names, the largest name groups - English names and Dutch-(pre)Modern names — dominate the table (see Table I).

\section{Lifestyles}

For each of twenty lifestyles, we have an average loading per name group. The higher a loading on a lifestyle, the more applicable the lifestyle is for that name group.

TABLE 2

TOP TWENTY OF NAMES PER CATEGORY OF TOTAL NUMBER OF YEARS OF EDUCATION OF THE PARENTS, WITH THE HIGHEST RANK ORDER PER NAME IN BOLD TYPEFACE

Total number of years of education of parents

\begin{tabular}{clllllll}
\hline & 8 primary & 11 vocational & 12 & 14 vocational & 14 & 17 vocational & 18+ \\
\hline 1 & Kevin & Kevin & Kevin & Tim & Tim & Tim & Thomas \\
2 & Patrick & Dennis & Kim & Kevin & Robin & Tom & Daan \\
3 & Danny & Mike & Dennis & Rick & Kevin & Sanne & Tim \\
4 & Wesley & Kim & Robin & Sanne & Tom & Anne & Anne \\
5 & Dennis & Rick & Mike & Kim & Kim & Daan & Laura \\
6 & leffrey & Patrick & Rick & Robin & Rick & Bart & Tom \\
7 & Samantha & Wesley & leroen & Jeroen & Nick & Bas & Lotte \\
8 & Chantal & Jeffrey & Mark & Nick & Sanne & Thomas & Bas \\
9 & Ricardo & Roy & Nick & Mark & Mark & Niels & Wouter \\
10 & Melissa & Nick & Danny & Lisa & Niels & leroen & Bart \\
11 & Mike & Robin & leffrey & Dennis & leroen & Sander & Sophie \\
12 & Wendy & Mark & Sanne & Mike & Laura & Robin & Sanne \\
13 & Kim & Danny & Roy & Niels & Lisa & Mark & Jasper \\
14 & Roy & Tim & Tim & Tom & Dennis & Kim & Max \\
15 & Linda & Jeroen & Patrick & Sander & Sander & Rick & Eva \\
16 & Joey & Chantal & Laura & Bas & Thomas & Lars & Niels \\
17 & Michael & Sanne & Sander & Laura & Bas & Lotte & Koen \\
18 & Nick & Stefan & Linda & Bart & Bart & Iris & Emma \\
19 & Mark & Melissa & Wesley & Lars & Iris & Laura & Martijn \\
20 & Robin & lordy & Jordy & Anouk & Mike & Martijn & Bram \\
\hline & & & & & & & \\
\hline
\end{tabular}


To summarize the relation between lifestyles and name groups, we applied a factor analysis (Gorsuch, I983) on the loading patterns. It showed that, on a few independent dimensions, the most important differences between the name groups in terms of lifestyle can be captured. Figure 5 shows the results on the first two dimensions, which already explain 75 percent of all variance in the lifestyle loadings.

The horizontal dimension in Figure 5 is governed by lifestyle differences that connect to income. Given the lifestyle labels assigned by WDM, a positive score indicates "well-to-do investors," "families that love traveling," "intensive users of the internet," while a negative score indicates "the financially limited" and "price conscious consumers." The vertical dimension indicates "fashionable young families" and "curious amusement searchers" at the positive side, while a negative score summarizes "socially concerned religious families."

The positioning of name groups on the horizontal dimension matches well with our previous results on family income and highest education. The vertical dimension seems to differentiate between philosophies of life, from conservative and religious to modern and trendy. The parents who chose Italian/Spanish or English names have a lower than average income, but are open to innovation and easily shed traditional values. The highest income groups favor Dutch, Hebrew, and Frisian names and are neither very modern nor very conservative. A conservative attitude is, not surprisingly, associated with more religious parents who opt for traditional names.

To demonstrate the lifestyle dimensions more directly, in Figure 6 we present the family income on the horizontal axis, and the probability of attending religious services on the vertical axis (inverse). The positions of the name groups in this figure match the lifestyle positions in Figure 5 quite well, although the dominantly Islamic parents from the Arabic and Turkish name groups have relatively high mosque attendance compared to their position in Figure 5.

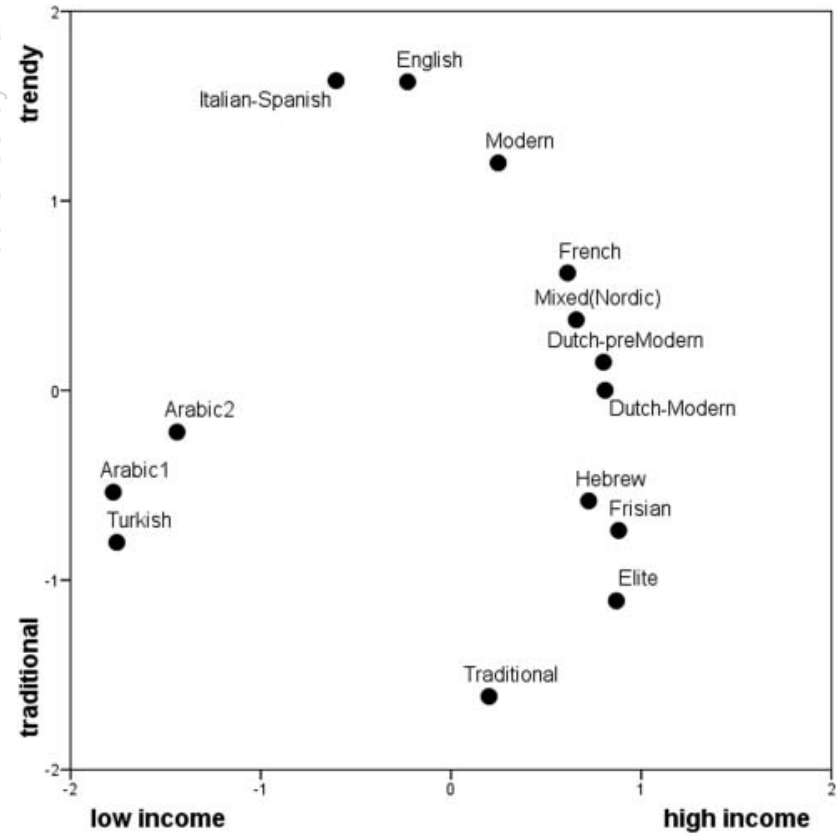

FIGURE 5 Name group positions on the two major lifestyle dimensions. The horizontal dimension is income-related, the vertical dimension differentiates between traditional and modern lifestyles. 


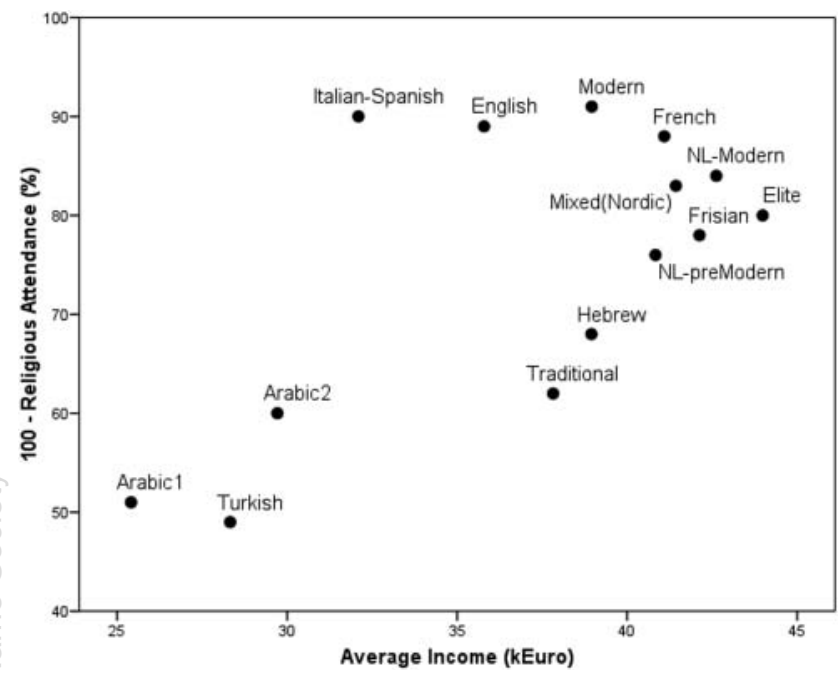

FIGURE 6 Family income of name groups versus religious attendance (inverse) of parents.

The probability of attending classical or pop concerts gives us comparable results, as can be seen in Figure 7. In this case, the income dimension seems to run diagonally from bottom left to upper right, since households with a low income cannot afford any concerts (although this may not be the only factor for the Arabic and Turkish parents), while for high-income households all options are open. The dimension orthogonal to income again differentiates between traditional and modern name groups: parents who favor traditional names have moderate but equal interests in classical and pop music, while parents who favor modern names are overwhelmingly interested in pop music only.

\section{Dynamics on the "lifestyle canvas"}

We investigated naming practices during a period of about one generation. This relatively long period has the advantage of a sufficient number of children for statistical analyses, but naming practice is by no means stable throughout such a period. Parents who had children in the I980s made other choices than parents twenty years later did. In the modern naming practice, most names have a life cycle: they emerge, become popular, and disappear again. The same pattern should be expected for name groups, which all were in some part of their life cycle during the period we have studied. There are name groups that are declining, while others are expanding (see tables in Bloothooft \& Groot, 2008). These dynamics in naming raise questions about the role of the socioeconomic factors involved. Is dynamic behavior limited to social strata, which each follow their own development, or are the dynamics much more complex, and involve diffusion of preferences through social classes?

It is possible to glimpse the answers to these questions by analyzing the largest twenty-eight name groups (of the original thirty-four) with respect to lifestyle loadings. The resulting two dimensions are shown in Figure 8. In addition to the more refined and complex presentation of the twenty-eight name groups, we indicate with an open triangle that a name group was declining between 1982 and 2005 , and by a filled triangle that it was expanding. 


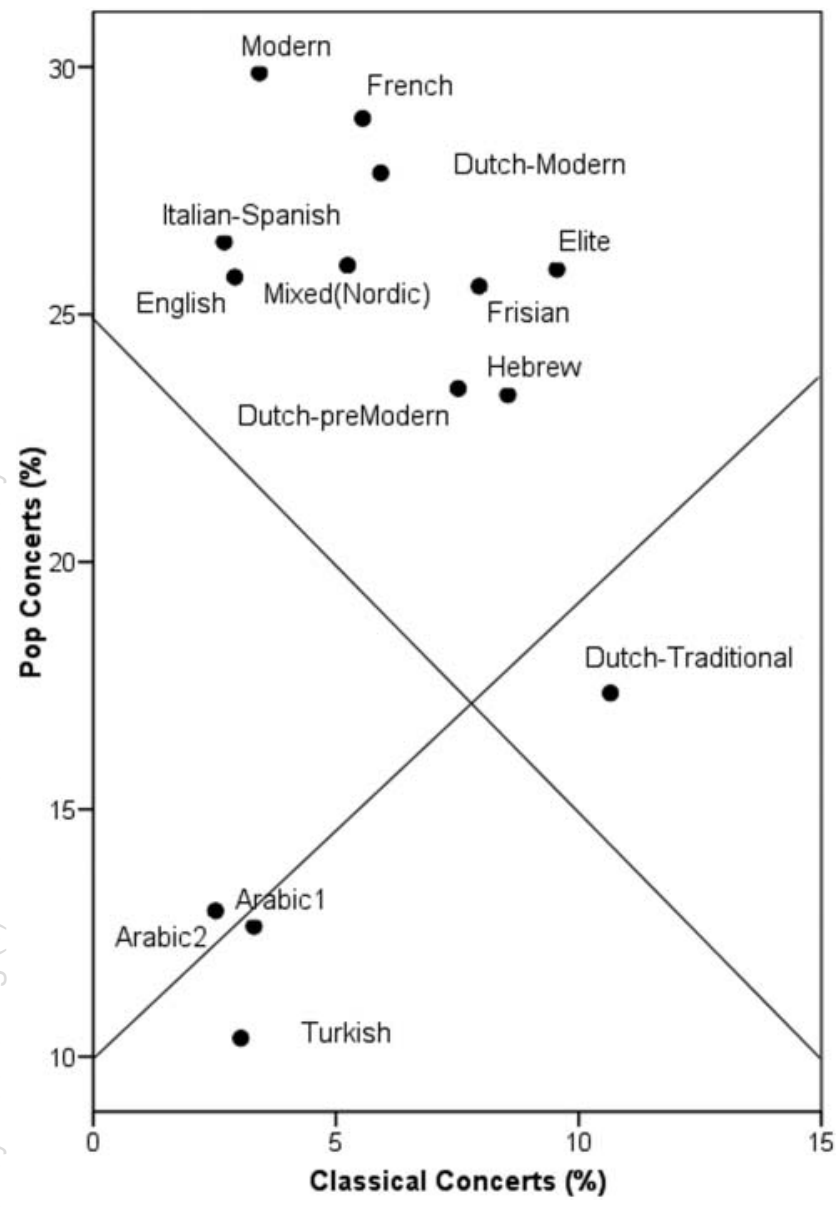

FIGURE 7 Attendance of classical and pop concerts by parents by name group. Rotated axes indicate a correspondence to the income/education dimension (starting left under), and the traditional-trendy dimension (starting right under).

The general composition of the name groups of course resembles the composition of the main name groups in Figure 5, but additional interesting details can be observed. At the top, we see that all English groups, both those in decline and expansion, are closely grouped together. This grouping suggests that the social classes that favor English names have their own dynamics, abandoning names that are oldfashioned (for this generation) and picking new ones. The exception to this generalization is the group of English royal names, which can be found at the bottom of the figure, together with other traditional groups that are all in decline. These consist of the two traditional Dutch name groups, one of the elite groups and a group with more traditional mixed Nordic names. As there will always be parents who wish to name their children after relatives, it is expected that the decline of these groups will end in stabilization, especially the traditional Dutch groups. In the right-hand part of the graph, the various elite, modern, Dutch, French, and mixed (Nordic) name groups cover a range along the conservative-trendy dimension, but still show convergence. The elite name groups group together, which can be interpreted as older elite parents choosing names in the I980s that were in decline over the whole period under study (Roderick and Liselotte), while younger elite parents now choose the up-and-coming 


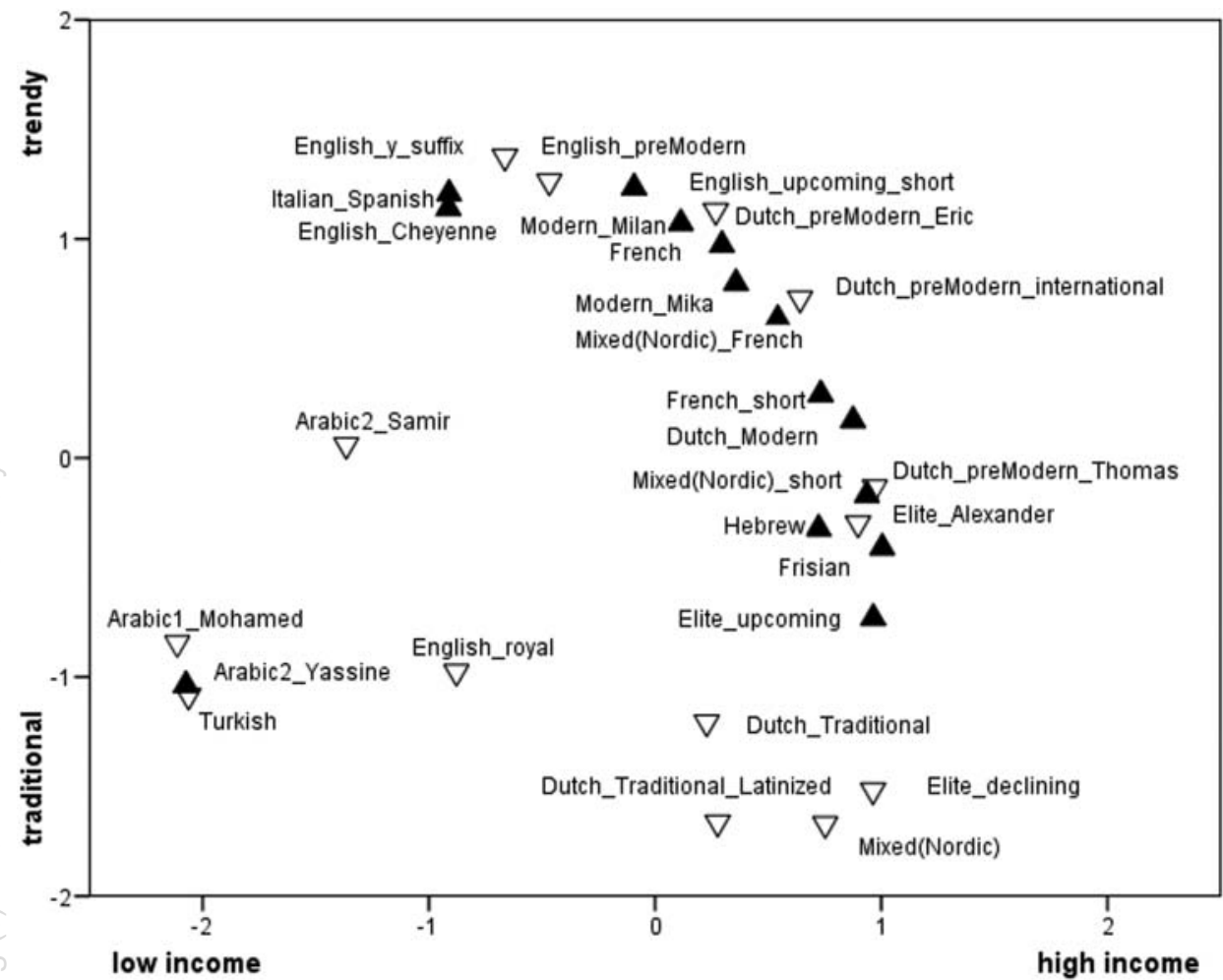

FIGURE 8 Positions of 28 more differentiated name groups in the plane of the two major lifestyle dimensions, associated with income (horizontal) and the traditional-trendy opposition (vertical). Open triangles indicate groups in decline, filled triangles designate expanding groups.

ones (Floris and Charlotte). In the social groups where older parents favored longer, pre-modern Dutch, and international names like Thomas, younger parents may opt for the increasingly popular groups of short Dutch Modern names (Tim and Sanne), Nordic short names (Mats and Bente), Hebrew (Daniël and Esther), or Frisian names (Jelle and Femke). These name groups can all be associated with parents that have a comparable lifestyle. The parents that favored shorter pre-modern international names like Mark, Peter, Paul, or Linda, may have successors that favored the up-andcoming French names (Thierry and Maxime), or modern names from the Mika and Milan groups. However, whether these interpretations are valid should be the subject of further research.

The overall picture is that declining name groups can be found grouped with corresponding expanding name groups nearby. This suggests that lifestyle, including underlying factors like education and income, provides a social canvas with local dynamics in naming practice. These dynamics may sometimes be very slow or even entirely absent, as we see with traditional Dutch names, which at present are mainly chosen by parents who still adhere to the practice of naming after relatives.

We did not examine whether individual name groups showed gradual changes from one lifestyle to another over time. Such changes could signal diffusion of name 
preferences among social classes, which could manifest in the movement of a name group across the "lifestyle canvas." There is little indication, however, that elite names will move through the whole spectrum of social strata into the classes that now favor English names, or vice versa. Diffusion of, for instance, Dutch, Frisian, Hebrew, and French names is nevertheless conceivable, given the noticeable spread seen in the top twenty of names per education category in Table 2.

\section{General discussion}

The relationship between the modern naming practice and socioeconomic determinants is fuzzy, but it does have some clearly identifiable characteristics. To establish this relationship in the full population, it is first necessary to identify the CEL groups in society, especially the groups that are associated with a closed community in which language and culture, including names, are highly valued for their role in identity. In our study, we identified the Arabic and Turkish name groups as unique CEL groups, distinguishing them from the others. Only after making this distinction can further analysis of socioeconomic determinants be pursued per CEL group. In the interpretation of results, it should be taken into account that all name groups were combined in a single analysis in this study.

We did not investigate all first names from the latest generation of children in the Netherlands, since the frequency of many names is too low for the name to be allocated to any name group in a statistically meaningful way. There is no doubt that the number of CEL groups in the Netherlands is comparable to the fifteen that have been identified for Great Britain (Mateos et al., 2007). Whereas the Arabic and Turkish name groups are easily identifiable and "closed" (since parents seldom choose a name outside these groups), the Surinamese and Antillean populations in the Netherlands show dominant preferences for, respectively, English and Spanish names. A complication is that these preferences are also shared by many Dutch parents, which makes a CEL group distinction between Antillean, Surinam, and Dutch parents impossible on the basis of first names only. The Surinamese and Antillean population, however, represents less than 2 percent of the total population, so their influence as specific CEL groups will not be very high, even if all names of their children are part of the very popular name groups.

Given the fuzzy nature of the relationship between naming practices and socioeconomic parameters, it is important to reduce uncertainties by extrapolating beyond the level of individual names, and using name groups defined by the shared name preferences of parents. Our hypothesis that parents who share name preferences also share socioeconomic determinants was supported, at least on a statistical basis. The variability among parents is very high, in that names from a name group could be found in almost all income and education categories, but clear tendencies are nevertheless visible from their distribution means. This observation supports the views of Desplanques (I986) and Lieberson (2000). The higher their education level and, correspondingly, family income, the more likely parents are to stick to Dutch names. These are not traditional names, but related abbreviated variants, or longer variants with historical connotations. In groups with lower income and education, there is a clear preference for non-Dutch names, likely influenced by the Anglo-American 
dominance of the popular media (Vandebosch, 1998). Although this is in line with the observations about naming practices in Western countries summarized in the introduction, we also identified another dimension, related to the philosophy and attitudes on life of the parents, conservative and traditional versus trend-sensitive. This dimension can be seen in many aspects of life: in fashion, in political preferences, and, as we showed, in the names of children. In this respect, parents' choices can be independent of education and income.

Our study only slightly touched upon the dynamics of naming practices, but our results raise many questions in this area. Modern naming practices imply a life cycle for names that seems to become increasingly shorter (Desplanques, I986), but our study suggests a more complex picture. The conservative-trendy dimension in particular appears to be associated with a differentiation in life-cycle duration, from a very long life cycle for traditional names to a very short one for name groups consisting of foreign and modern names. If names have a life cycle, though, what about the life cycle of a name group? It is unlikely that all names in a group become popular simultaneously, and remain popular for exactly the same period of time. Therefore name groups are likely to show a more gradual rise and decline in popularity than individual names do. Various models for the life cycle and diffusion of name groups are conceivable. We showed that name groups are related to social class indicators. Over time, as the preferences of a new generation of parents emerge, new names may replace old ones in a particular social class. In an analysis that covers a generation, as ours, a shift in preferences may show up in two name groups, one in decline the other on the rise. Another possibility, however, is that an analysis window of one generation is too short to disentangle names on the rise and in decline and that they merge into a single name group. It is also conceivable that a name group might migrate during its life cycle from one social class to another, or even from one CEL group to another. In such a case, this could show up as a longer name group life cycle with multiple peaks. Finally, it is possible that a name group disintegrates over time, with some names being abandoned by one social class (or CEL group) and emerging in another. This possibility would manifest as multiple peaks in the popularity distribution of the relevant individual names. It would be exciting to demonstrate one or more of these processes to support the view that a simple top-down diffusion model is not sufficient to describe the social patterns in naming practices over the last century.

\section{Acknowledgment}

We wish to thank WDM company, and especially Bob Hoogewind, for making available the data from De Grote Consumenten Enquête.

\section{Bibliography}

Andersen, Christian. 1977. Studien zur Namengebung in Nordfriesland. Die Bökingharde I760-1970. ClausthalZellerfeld: Böneke.

Besnard, Philippe \& Cyril Grange. I993. "La fin de la diffusion vertigale des gouts? (Prénoms de l'elite et du vulgum)." L'Année sociologique 43: 269-94. 
Bloothooft, Gerrit, Emma van Nifterick, \& Doreen Gerritzen. 2004. On First Names - How The Netherlands Gets its First Names. Utrecht: Het Spectrum (in Dutch).

Bloothooft, Gerrit \& Loek Groot. 2008. "Name Clustering on the Basis of Parental Preferences.” Names 56: III-63.

Debus, Friedhelm, Joachim Hartig, Hubertus Menke, \& Günter Schmitz. I973. "Namengebung und soziale Schicht. Bericht über ein Projekt zur Personennamenkunde." Naamkunde 5: 368-405.

Desplanques, Guy. I986. "Les enfants de Michel et Martine Dupont s'appellent Nicolas et Céline." Economie et statistique I84(I): $63-83$.

Dupâquier, Jacques, Jean-Pierre Pélissier, \& Danièle Rébaudo. I986. Le temps des Jules: les prénoms en France an XIXe siècle. Paris: Ed. Christian.

Fryer, Roland G. \& Steven D. Levitt. 2004. "The Causes and Consequences of Distinctly Black Names." The Quarterly Journal of Economics II9(3): 767-805.

Gerhards, Jürgen. 2003. Die Moderne und ihre Vornamen, eine Einladung in die Kultursoziologie. Wiesbaden: Westdeutscher Verlag.

Gorsuch, Richard L. I983. Factor Analysis. New Jersey: Erlbaum.

Leys, Odo. I974. "Sociolinguistic Aspects of Namegiving Patterns." Onoma I8: 448-55.

Lieberson, Stanley \& Eleanor O. Bell. I992. "Children's First Names: an Empricial Study of Social Taste." The American Journal of Sociology 98(3): 5II-554.

Lieberson, Stanley. 2000. A Matter of Taste-How Names, Fashions and Culture Change. New Haven: Yale University Press.

Mateos, Pablo, Richard Webber, \& Paul Longley. 2007. "The Cultural, Ethnic, and Linguistic Classification of Populations and Neighbourhoods Using Personal Names." CASA Working Paper iı6. Available at: <http://www.casa.ucl.ac.uk/working_papers/paperıi6.pdf>

National Bureau of Statistics, The Netherlands. 2007. Available at: < $\underline{w w w . c b s . n l}>$

Seibicke, Wilfried. I996. "Traditionen der Vornamengebung. Motivationen, Vorbilder, Moden: Germanisch." Name Studies: An International Handbook of Onomastics, Namenforschung in Handbook of Onomastics. Ed. E. Eichler, G. Hilty, H. Loffler, H. Steger, L. Zgusta, and W. Bright. Berlin-New York: De Gruyter, I207-I4. Vandebosch, Heidi. I998. "The Influence of Media on Given Names.” Names 46: 243-62.

\section{Notes on contributors}

Gerrit Bloothooft is researcher at the Utrecht Institute of Linguistics. His interests are in language technology and onomastics. He has published on issues of nominal record linkage and was co-author of a landmark book on first names in the Netherlands. He recently launched online full population databases of first names and family names in the Netherlands.

Correspondence to: Gerrit Bloothooft, Utrecht institute of Linguistics - OTS, Utrecht University, Trans ıо, 35 I2 JK Utrecht, The Netherlands. Email: g.bloothooft@uu.nl

David Onland is research assistant. He studied Computer Science and History and is working on his MA in political and cultural History. 